\title{
Development of a Near-Field Magneto-Optical Microscopy for Studying Ultrafast Magnetization Dynamics
}

\author{
G. Zoriniants ${ }^{1}$, D. Englund ${ }^{1}$, O. Kurnosikov ${ }^{1}$, C.F.J. Flipse ${ }^{1}$, \\ E. Riedo ${ }^{2}$, H. Brune ${ }^{2}$, W.J.M. de Jonge ${ }^{1}$ and B. Koopmans ${ }^{1}$ \\ ${ }^{1}$ Eindhoven University of Technology, P.O. Box 513, 5600 MB Eindhoven, The Netherlands \\ ${ }^{2}$ Ecole Polytechnique Federale de Lausanne, IPN, 1015 Lausanne, Switzerland
}

\begin{abstract}
Apertureless magneto-optical near-field microscopy is developed for studying subpicosecond spindynamics at nanometer spatial scale. Polarization modulation and tip vibrations are implemented. Polarization responsivity of the tip-induced scattering is demonstrated using polarization modulation and tip vibrations. A near-field magneto-optical contrast is achieved for a thin ferromagnetic film with in-plane magnetization; however, its interpretation is shown to be nontrivial. Distribution of the evanescent field is measured vs. tip-sample distance. Interference of the near-field with the far-field scattered light is found at distances ca. $300 \mathrm{~nm}$ and more, and strong tip-surface interaction that quenches the detected scattering is found at distances below 20 $\mathrm{nm}$.
\end{abstract}

\section{INTRODUCTION}

The study of magnetic micro- and nanostructures and local magnetic excitations in laterally homogeneous systems is an intriguing novel issue. An absolute requirement for the exploration of spin dynamics of these systems is the development of novel experimental techniques that combine high spatial resolution (towards the nanometer scale), high spin sensitivity, and high temporal resolution (down to picoseconds).

We present a technique that implements magneto-optical (MO) detection (very efficient for ultrafast spin dynamics measurements) in near-field microscopy ${ }^{\text {i-iv }}$ using an apertureless probe. ${ }^{\text {ii, iv }}$ A metallic or dielectric tip converts the near-field into propagating waves, which are then detected in the far field. Local magnetization of a sample produces variations of the polarization state of the detected light. To resolve the small Kerr or Faraday effect, we use polarization modulation by a photo-elastic (PEM) or electro-optical (EOM) modulator and lock-in detection.

The technique has several crucial features that makes it applicable for measurements of ultrafast spindynamics with nanoscale spatial resolution. Compared to fiber-optic SNOM, the polarization state of light can be much better determined, so that smaller Kerr or Faraday effects can be measured. The absence of fiber optics allows for short laser pulses down to a femtosecond scale. Metallic tips have a high efficiency for converting the evanescent field to propagating waves, ${ }^{\mathrm{iv}}$ thus allowing for a smaller tip size and, accordingly, higher spatial resolution. In addition, metallic tips are more thermostable, so high energy light pulses may be applied for time-resolved studies.

In this paper several issues of the apertureless MO-SNOM are discussed. The spatial mapping of an evanescent field is demonstrated. Clearly distinct patterns are observed for the intensity and polarization across the focused laser spot. A proof-of-principle for the resolution of the MO scattered signal for a 5 $\mathrm{nm}$ cobalt film with in-plane magnetic field is presented. However, an unambiguous identification of

CP696, Scanning Tunneling Microscopy/Spectroscopy and Related Techniques: 12th International Conf., edited by P. M. Koenraad and M. Kemerink

(C) 2003 American Institute of Physics 0-7354-0168-3/03/\$20.00 
the near-field character is nontrivial because of the interference of tip-induced scattering with scattering from irregular sample defects.

We performed a careful study of the evanescent field decay by varying the tip-sample distance. The evanescent field, in general, decays exponentially as a function of distance to the surface. At very low distances (ca. $20 \mathrm{~nm}$ ) the tip-surface electromagnetic interaction is found to lead to quenching of the detected scattering; at distances comparable to the wavelength, near- and far-field interference occurs.

\section{EXPERIMENT}

As in all other scanning probe microscopies, the core of the set-up is a tapered tip and a system for its precise positioning over a sample. Numerous distance control mechanisms are used in AFM, STM and SNOM, and accordingly different shapes and materials of tips are used. In principle, many of these are potentially applicable for MO SNOM. Part of the results presented in this paper were obtained with a commercial AFM (Park Scientific Autoprobe) equipped with dielectric tips and operating in noncontact mode. The set-up was adapted for optical access. Based on the experience with this set-up, a novel dedicated and home-built system is being developed, especially designed for the MO SNOM experiment. The latter system is based on shear force detection for feedback. We will refer to the two systems as 'AFM' and 'shear force', respectively.

The general scheme of the 'shear force' set-up is shown in Figure 1. Thin films of ferromagnetic materials are placed on the top of a glass hemisphere. The light from a $5 \mathrm{~mW} \mathrm{He}-\mathrm{Ne}$ laser $(638 \mathrm{~nm})$ is directed at the sample surface from the glass side in total internal reflection mode. We use tungsten tips made by electrochemical etching (the same procedure is used in STM). The apex radius was smaller than $50 \mathrm{~nm}$ (measured by scanning electron microscopy); the tips showed high mechanical and chemical stability. A further advantage of a metallic tip is that the interaction with the evanescent field and therefore the scattering of light to be detected is stronger than for dielectric materials .v

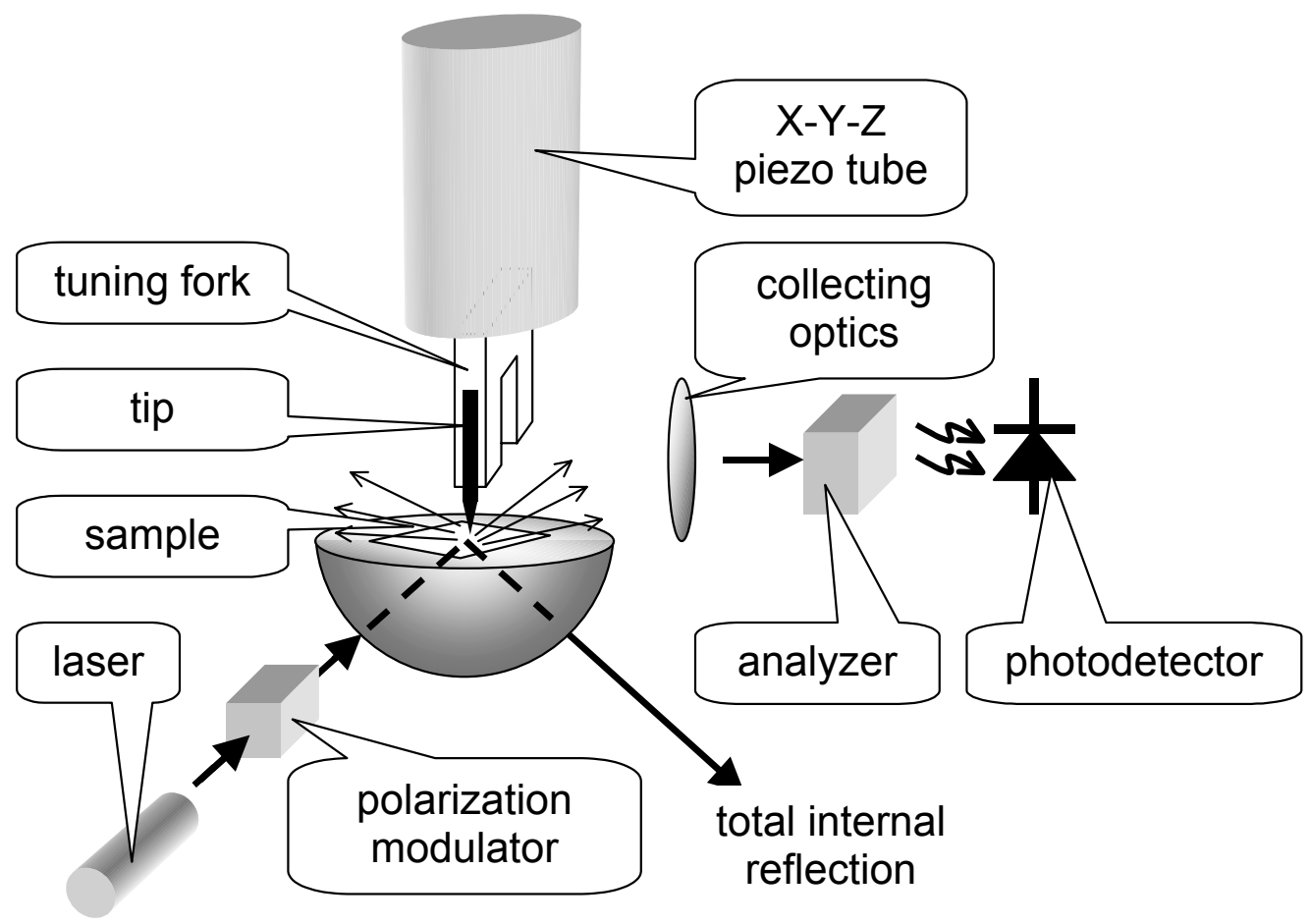

FIGURE 1. Schematic of the apertureless near-field magneto-optical microscope with shear-force detection of tip-sample distance. 
We have chosen very small tuning forks (Seiko Epson corp., "leg" dimensions $2.7 \times 0.8 \times 0.1 \mathrm{~mm}^{3}$ ) and a tiny tungsten wire of diameter $0.05 \mathrm{~mm}$. The fork was operated at its resonance frequency around $32 \mathrm{kHz}$. This value was slightly different from the factory setting due to the presence of the tip. The Qvalue of the resonator was tuned by varying the tip size and the amount of glue. It was chosen around 500 as a result of trade-off of high sensitivity against fast response.

The performance of the shear-force feedback system was tested on model samples. Vertical resolution was about $5 \mathrm{~nm}$; lateral resolution was better than $100 \mathrm{~nm}$. The set point of feedback corresponded to 5\% amplitude damping. Topographical noise was less than $1 \mathrm{~nm}$ and was mostly of mechanical or acoustical origin.

A confocal optical scheme was applied to collect the scattered light by a photodiode and at the same time monitor the tip and sample by a CCD camera. The total optical magnification of the detection scheme was about x20 and the resolution was about $3 \mu \mathrm{m}$. To separate the near-field component from the total light scattered from the sample defects, we modulated the tip-sample distance $z$ by additional piezo stack. Only the contribution of the scattering by tip was detected, because it is the only signal depending on the tip height. Thus, for small modulation amplitudes, the detected optical signal was proportional to the $z$-derivative of the evanescent field intensity and to the amplitude of $z$-modulation.

To perform the magneto-optical measurements, we used a broadband electro-optical phase modulator (New Focus Model 4002M). The configuration of the detection optics is designed in such a way to measure the in-plane components of the sample magnetization, which is very interesting from the practical point of view.

The 'AFM system' differed in some points. A ninety degrees prism was used, to which a lens was glued with index matching fluid to reduce diffraction effects. Polarization modulation was achieved by a photoelastic modulator (PEM), operating at a fixed frequency of $\mathrm{f}_{\mathrm{PEM}}=50 \mathrm{kHz} . \mathrm{Si}_{3} \mathrm{~N}_{4}$ tips with a radius of $20 \mathrm{~nm}$ were used, at a resonance frequency around $\mathrm{f}_{\mathrm{AFM}}=60 \mathrm{kHz}$. Scattered light was detected with a photomultiplier tube, collecting light at an azimuthal angle of $45^{\circ}$ with respect to back scattering, and at a polar angle of $55^{\circ}$ with respect to the surface normal.
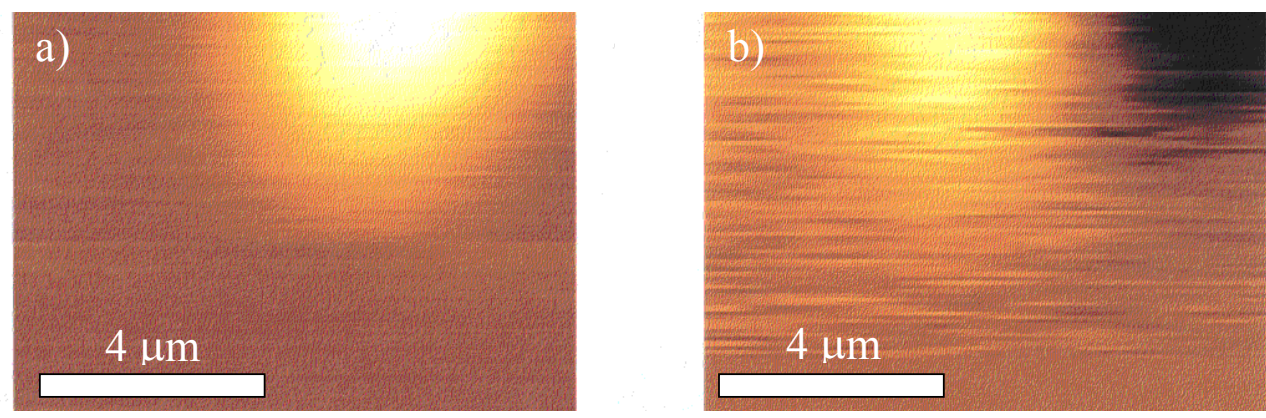

FIGURE 2. Image of focused laser spot; a) intensity , b) polarization rotation.

\section{RESULTS AND DISCUSSION}

\section{Imaging of the laser spot, polarization and MO sensitivity}

With the 'AFM system' we verified the polarization and MO sensitivity, using a thin ferromagnetic cobalt film of $7 \mathrm{~nm}$ on a glass substrate, mounted with index-matching fluid on the prism. Figure 2 displays the scattered light intensity (a) and polarization (b), recorded while the vibrating AFM tip scanned the sample in non-contact mode. It was verified that the topography image revealed no significant features across the scanned area. Both optical images were obtained by using a lock-in amplifier that filtered out the signal at $2 \mathrm{f}_{\mathrm{PEM}}-\mathrm{f}_{\mathrm{AFM}}$. Selecting the second-harmonic of the PEM frequency means that the rotation of the light, rather than the ellipticity, is measured. Setting the orientation of the analyzer at $45^{\circ}$ with respect to the main axis of the PEM selects a signal that is mostly 
sensitive to the intensity (Fig. 2 (a)), whereas placing it parallel to the PEM selects the true polarization signal (Fig. 2 (b)). A striking contrast between the laser spot profiles in the two modes is observed. The intensity image displays an ellipsoidal profile, determined by the diffraction limited spot size of $\sim 4 \mu \mathrm{m}$. The polarization image is completely different, and shows a positive and a negative lobe. The latter can be understood from the variation in incident and scattering angle while scanning across the laser spot.

Next, we carefully measured the change of the polarization signal upon reversal of the magnetization of the ferromagnetic film. Figure 3 displays a well resolvable change in polarization after reversing the magnetization by an in-plane field pulse of $\sim 300 \mathrm{Oe}$, generated by an electro-magnet mounted near the AFM tip. Note that data are acquired in remanence, i.e., in the absence of a magnetic field.

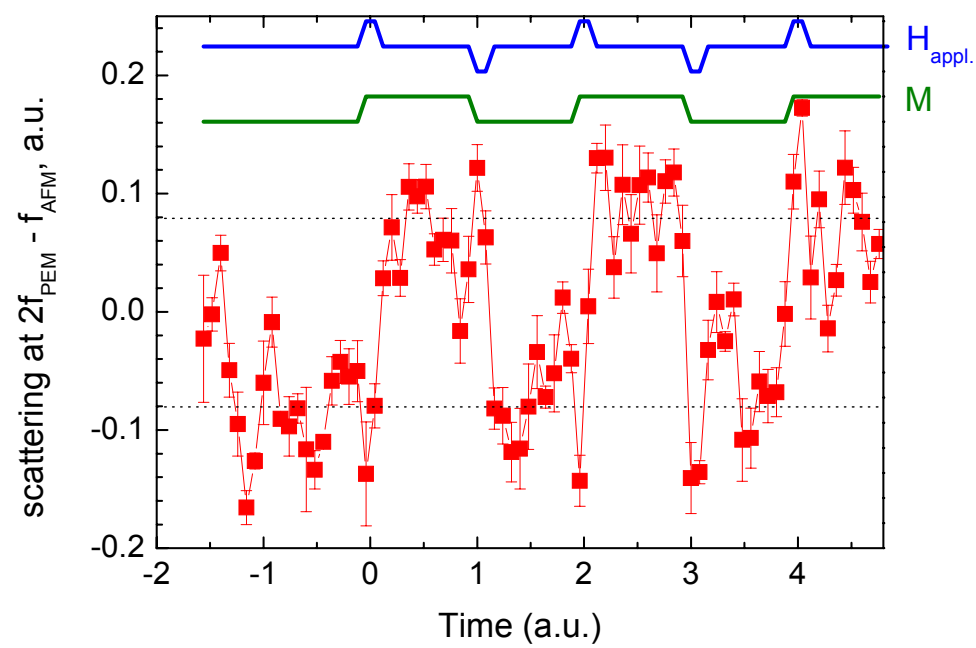

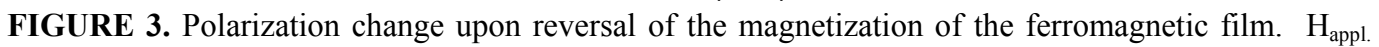
denotes a schematic plot of external field applied, and $\mathrm{M}$ is a schematic plot of film magnetization.

The images could not be interpreted easily. Most importantly, the signal in the geometry employed can be described as:

$$
\mathrm{I} \propto\left|\mathrm{E}_{\mathrm{s}}\right|^{2}+\mathrm{E}_{\mathrm{s}} \cdot \mathrm{E}_{\mathrm{t}}(\vec{r}, d) \cdot \exp (\overrightarrow{\mathrm{i}} \vec{q} \cdot \vec{r})+\left|\mathrm{E}_{\mathrm{t}}(\vec{r}, d)\right|^{2}
$$

where $\mathrm{d}$ is the tip-sample distance, $\vec{r}$ denotes the in-plane position of the tip across the sample, $\vec{q}$ is the scattering vector, $E_{t}$ is the tip-induced scattering (in the far field) and $E_{s}$ represents the sum of scattering from all non-tip related irregularities across the spot. Note that the latter is independent of the tip position, and, as one expects, may be easily corrected for. However, we explicitly verified that $\mathrm{E}_{\mathrm{s}}$ depended on the orientation of the magnetization via the MO Kerr effect, by recording the polarization contrast after overlapping the laser spot with a strongly scattering feature and completely withdrawing the tip. Thus, features in the MO-SNOM scan can potentially be the result of the second term in Eq. (1), being the product of a magnetization-dependent $E_{s}$ and a tip-position dependent $E_{t}$. So far we were not able to complete the explicit proof that $\mathrm{E}_{\mathrm{t}}$ depends on the magnetization.

We believe that once the final proof has been made, the extension of the technique to time-resolved approaches will be relatively easy. In particular, it is well known that time-resolved MOKE within a pump-probe scheme is typically two to three orders more sensitive than static MOKE, because of the higher rejection of mechanical instabilities and insignificance of topography-related polarization effects.

\section{Near-Field Spatial Distribution}

If light passes from one medium to another of lower refractive index (e.g., from glass to vacuum), then if the incidence angle $i$ exceeds the critical angle $i_{\mathrm{o}}=\arcsin 1 / \mathrm{n}(\mathrm{n}$ is the relative refractive index), all light is reflected, and no refraction occurs. The wavenumber of this beam is imaginary in this case. 
This means that there are no refracted propagating waves, but instead the electromagnetic field decays exponentially:

$$
E_{t} \propto \exp \left(-\frac{2 \pi z g}{\lambda}\right), g=\sqrt{\left(\frac{\sin i}{\sin i_{0}}\right)^{2}-1},
$$

where $z$ is the distance from the interface, $\lambda$ is the wavelength of light in the referred medium, and $g \approx 0.83$ for the geometry of our set-up. It is this "near-field" that is picked up by the SNOM tip.

We can assume that the dipolar moment of the tip is induced by the near-field and is proportional to its strength, and therefore the intensity of the detected light must be proportional to the $\left|E_{t}\right|^{2}$. So, the detected SNOM signal must decay exponentially with tip-sample distance with the factor $\lambda / 4 \pi g$.

We measured this distance dependency by approaching and retracting the tip from the surface. In general, an approximately exponential trend is reproduced in all experiments. Surprisingly, however, in specific cases the measured curves deviate significantly from the exponential law, which is best seen at large $(>300 \mathrm{~nm})$ and small $(<20 \mathrm{~nm})$ tip-sample distances.

In Figure 4 we present a typical example of $z$-dependency of the scattering intensity, which was taken when the sample exhibited significant extra scattering from surface defects. A clear deviation from the exponential law is seen.

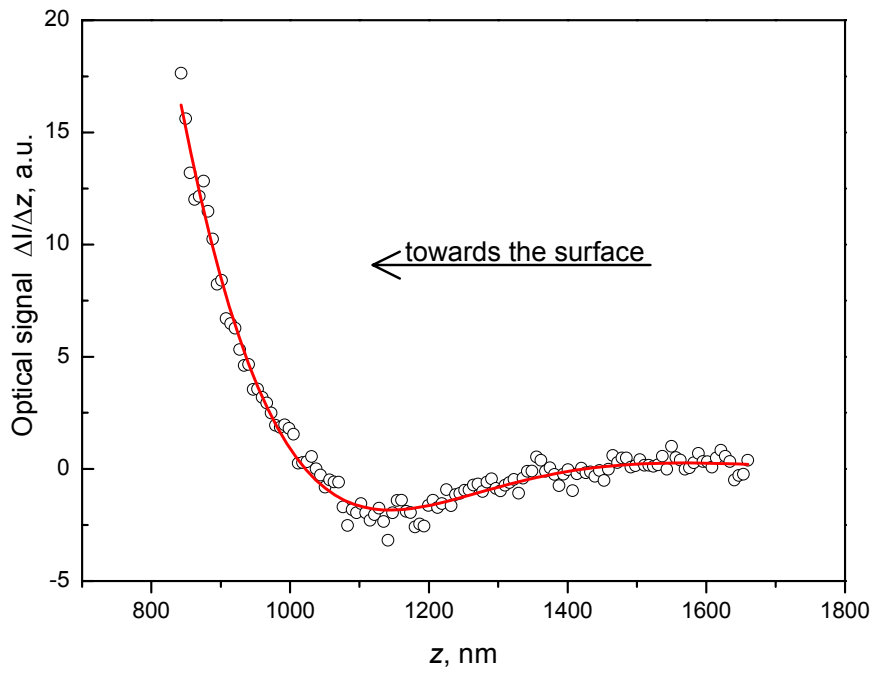

FIGURE 4. Dependency of the apertureless SNOM signal on the tip-sample distance. Open circles experimental data points, solid curve - fitting result. The $z$-axis has an arbitrary offset.

To explain the long-distance deviation, we assumed the presence of other scattering center(s) on the sample surface. In addition to the near-field re-emitted light by the tip $E_{t}$, a far-field wave from these scattering centers $E_{s}$ appears. The interference of the two gives the total field:

$$
E=E_{t}+E_{s}=A \exp (-2 \pi g z / \lambda)+B \exp (i 2 \pi z / \lambda+i \varphi),
$$

where $\mathrm{A}$ and $\mathrm{B}$ are amplitudes of the two waves and $\varphi$ is the relative phase. We derived the expression for the light intensity from (3) and found the best-fit curve by a least-squares method (Figure 4). The excellent coincidence of the calculated curve with the experimental data suggests that the contribution of the far-field light cannot be ignored in future experiments. Besides, the extracted relative amplitudes and phases of the near- and far-fields $(A / B=-4.3, \varphi=0.07)$ can provide additional information on the sample properties.

The far-field light sources are induced by some surface defects that are fixed somewhere on the sample plain. This part of the optical signal is not changing during $x-y$ scanning. Of course, it would be 
favorable to diminish this contribution as much as possible. In principle, this long-distance minimum can be avoided by finding a defect-free sample site, but generally it appears in most of our measurements, especially for samples with strong scattering.

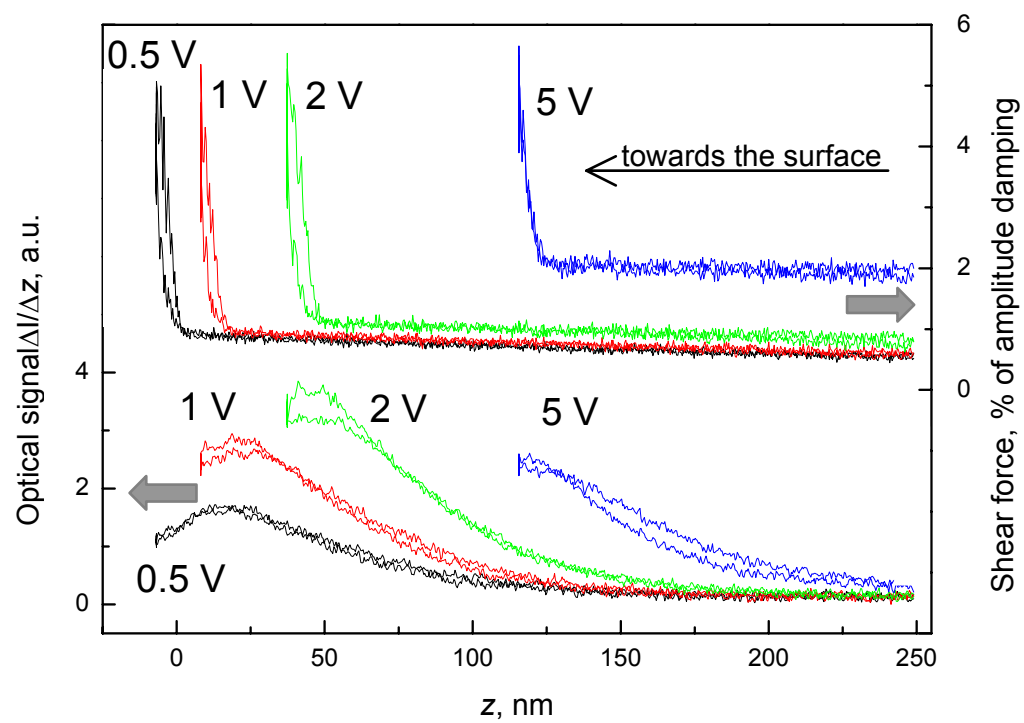

FIGURE 5. Dependency of the apertureless SNOM signal intensity on the tip-sample distance. The upper set of curves is the signal from the shear-force detector (right axis); the lower set of curves is the optical signal (left axis). The voltages indicate the amplitudes of modulation of the tip-sample distance. The $z$-axis has an arbitrary offset.

The other deviation from the exponential law occurs at smaller $z$, typically under $20 \mathrm{~nm}$. It is also very sensitive to a particular site of the sample and the shape of the tip. Figure 5 shows the $z$ dependence of the optical signal taken with several different amplitudes of $z$-modulation. The amplitude of $z$-modulation is proportional to the voltage applied to the driving piezo that is shown in Figure 5. The upper curves show the moment when the tip starts to touch the surface. From these data, we can estimate the coefficient between driving voltage and $z$-modulation amplitude to be $\sim 30 \mathrm{~nm} / \mathrm{V}$.

It is clearly seen, especially for the $0.5 \mathrm{~V}$ and $1 \mathrm{~V}$ curves, that the deviation of the optical signal from exponential starts well before touching the surface, i.e. when there is still no change of the shear-force signal. Thus we can exclude any artifacts related to the change of $z$-modulation amplitude in the proximity of the surface. The quenching of the signal that we see is truly optical.

This local decrease of the optical signal can be interpreted in terms of surface-induced quenching of the dipolar emission from the tip. ${ }^{\mathrm{vi}}$ Indeed, when an electric dipole is brought close to a metallic or a dielectric surface, it creates an electrostatic image that acts as a dipole of opposite direction, which makes the total electric field from the two dipoles weaker. In the extreme, when the image dipole is equal and opposite to the original one, they form a quadrupole, and no dipolar emission can be observed. This effect should be strongly dependent on the surface and tip properties. So, unlike the simple exponential decay, the small-distance deviation can be used for more specific analysis of the material properties.

\section{CONCLUSIONS}

We designed and built an experimental set-up that combines the advantages of near-field magnetooptical microscopy with the possibility to perform sub-picosecond studies of magneto-dynamics. Major 
parameters of the microscope were tested; its resolution and optical efficiency were examined using model samples.

The spatial mapping of an evanescent field was performed. Clearly distinct patterns were observed for the intensity and polarization across the focused laser spot. Magneto-optical responsivity was shown for cobalt films in remanence. This proved the feasibility of the MO-SNOM technique for detecting the in-plane magnetic moment of ferromagnetic thin films.

Having discussed the basic operation of our shear force system, we addressed another relevant issue for the apertureless approach: a fundamental study of the dependence of the scattered light intensity on tip-sample distance. Significant deviations from the simple exponential decay were observed. At distances above $300 \mathrm{~nm}$ from the sample, interference of the evanescent and propagating fields was seen. At distances less than $20 \mathrm{~nm}$, strong tip-sample interaction was found to lead to the signal decrease. This interaction may be explained in terms of induced dipolar images and should be strongly dependent on the sample and tip properties.

\section{ACKNOWLEDGMENTS}

This work is part of the research program of the "Stichting voor Fundamenteel Onderzoek der Materie (FOM)", which is financially supported by the "Nederlandse Organisatie voor Wetenschappelijk Onderzoek (NWO)". Kees Flipse acknowledges his stay in laboratory of Prof. Klaus Kern in Lausanne where the work has started.

\section{REFERENCES}

i. E. Betzig, J. K. Trautman, R. Wolfe et al., Appl. Phys. Letters 61, 142-144 (1992).

ii. T.J. Silva, S. Schultz, D. Weller, Appl. Phys. Letters 65, 658-660 (1994).

iii. P. Fumagalli, A. Rosenberger, G. Eggers et al., Appl. Phys. Letters, 72, 2803-2805 (1998).

iv. H. Wioland, O. Bergossi, S. Hudlet et al., Eur. Phys. J. AP, 5, 289-295 (1999).

v. R.E. Larsen, H. Metiu, J. Chem. Phys., 114, 6851-6860 (2000).

vi. J. A. Porto, P. Johansson, S. P. Apell, T. López-Ríos, Phys. Rev. B, 67, 085409 (2003). 